\title{
Synchronized Formation and Remodeling of Postsynaptic Densities: Long-Term Visualization of Hippocampal Neurons Expressing Postsynaptic Density Proteins Tagged with Green Fluorescent Protein
}

\author{
Tatsuhiko Ebihara, ${ }^{1}$ Izumi Kawabata, ${ }^{2}$ Shinichi Usui, ${ }^{3}$ Kenji Sobue, ${ }^{3}$ and Shigeo Okabe ${ }^{1,2,4}$ \\ ${ }^{1}$ Molecular Neurophysiology Group, Neuroscience Research Institute, National Institute of Advanced Industrial Science and Technology, Ibaraki 305-8566, \\ Japan, ${ }^{2}$ Department of Anatomy and Cell Biology, School of Medicine, Tokyo Medical and Dental University, Tokyo 113-8519, Japan, ${ }^{3}$ Department of \\ Neuroscience, Osaka University Graduate School of Medicine, Osaka 565-0871, Japan, and ${ }^{4}$ Core Research for Evolution Science and Technology, Japan \\ Science and Technology Corporation, Kawaguchi 332-0012, Japan
}

To explore mechanisms governing the formation and remodeling of postsynaptic density (PSD), we used dissociated cultures of hippocampal neurons isolated from transgenic embryos expressing green fluorescent protein (GFP)-tagged PSD proteins PSD-Zip45 (Homer 1c) and PSD-95. Expression of GFP-tagged PSD molecules was stable, and the remodeling process of PSDs could be followed for $>1$ week. A higher expression level of GFP-PSD-Zip45 enabled us to quantitatively analyze the amount of PSD-Zip45 clusters during development. Repetitive imaging of the same cell populations between 11 and $17 \mathrm{~d}$ in culture revealed an increase of the average PSD-Zip45 cluster density from 0.32 to $0.73 / \mu \mathrm{m}$. Newly generated dendrites rapidly acquired GFP-PSD-Zip 45 clusters, and their density reached the level of parental dendrites within a few days. Temporal profiles of GFP-PSD-Zip45 cluster density showed a variety of patterns. Some dendrites showed a monotonous increase of clusters, whereas others showed complex patterns, including short decremental stages. Analysis of long-term remodeling of PSD-95-GFP clusters confirmed that the decremental stages were not specific to the PSD-Zip45 clusters. Comparison of the temporal profiles of the cluster density among neurons indicated synchronization of both GFP-PSD-Zip45 and PSD-95 clustering within individual cells. Furthermore, activation of cAMP-dependent protein kinase suppressed the decremental stages of cluster remodeling. These observations suggest the presence of signaling mechanisms that can induce synchronized addition or elimination of PSD proteins throughout dendritic arborization of a single neuron.

Key words: postsynaptic density; green fluorescent protein; fluorescence microscopy; hippocampus; homer; transgenic mouse

\section{Introduction}

A major functional role of the dendrite is to form a synaptic junction with incoming axons and to integrate the activity of individual synapses. The number and distribution of synaptic contacts on dendrites should be regulated by both extracellular signals and intracellular mechanisms (Scott and Luo, 2001). Although candidate molecules that influence synapse formation have been identified, little is known about the mechanisms controlling the number and distribution of synapses along dendritic arborization. Does synapse density increase monotonously in development? Do newly generated and preexisting dendrites have similar or distinct functions in synaptogenesis? To what extent do dendrites actively regulate synapse number? Alternatively, do dendrites function as mere receptive structures? To answer these types of questions, it is necessary to obtain a more complete view

Received June 13, 2002; revised Dec. 17, 2002; accepted Dec. 23, 2002.

This work was supported by grants from the Ministry of Education, Science, Sports, Culture and Technology of Japan, the Core Research for Evolutional Science and Technology of Japan Science and Technology Corporation, and the Human Frontier Science Program. We thank H. Okado for the generation of recombinant adenoviruses and Y. Kanegae and I. Saito for materials used in adenovirus construction.

Correspondence should be addressed to Shigeo Okabe, Department of Anatomy and Cell Biology, School of Medicine, Tokyo Medical and Dental University, 1-5-45, Yushima, Bunkyo-ku, Tokyo 113-8519, Japan. E-mail: okabe.cbio@tmd.ac.jp.

Copyright $\odot 2003$ Society for Neuroscience $\quad 0270-6474 / 03 / 232170-12 \$ 15.00 / 0$ of how individual synapses are added and eliminated during the period of synaptogenesis and synapse remodeling.

Recent technical advances in imaging techniques in combination with the generation of a variety of green fluorescent protein (GFP)-tagged synaptic proteins have revealed the dynamics of synaptic structure and turnover of proteins localized at synapses (Okabe et al., 1999; Shen and Meyer, 1999; Ahmari et al., 2000). GFP-tagged synaptic molecules were introduced into cultured neurons by transfection methods or virus-mediated gene transfer techniques. Although these studies provided information on synapse remodeling in time scales from seconds to hours, prolonged observation of single neurons over days or weeks was not possible. This difficulty was mainly derived from the fact that the expression level of GFP-tagged molecules introduced by either transfection or virus-mediated gene transfer was variable among individual cells and unstable over a time scale of days. To understand the basic mechanisms of long-term synaptic remodeling, it is necessary to devise a method to achieve stable expression of GFP-tagged synaptic proteins.

Postsynaptic density (PSD) is a thickening of the postsynaptic membrane of excitatory synapses in the CNS. The PSD contains a variety of neurotransmitter receptors, signaling molecules, and anchoring-scaffolding proteins composed of distinct proteininteracting domains (Kim and Huganir, 1999; Kennedy, 2000; 
Sheng and Sala, 2001). PSD-95/SAP90 and PSD-Zip45 (Homer $1 \mathrm{c} /$ Vesl-1L) are major PSD proteins that are localized to the excitatory postsynaptic sites in the hippocampus. PSD-95 is a protein with a multidomain structure and binds with other postsynaptic molecules, including NMDA receptors. PSD-Zip45 belongs to the Homer/Vesl protein family and binds to both group I metabotropic glutamate receptors $1 / 5$ (mGluR1/5) and $\mathrm{IP}_{3}$ receptors (Brakeman et al., 1997; Kato et al., 1998; Sun et al., 1998; Xiao et al., 1998; Tadokoro et al., 1999). Analysis of these two PSD proteins tagged with GFP revealed the distinct dynamic properties (Okabe et al., 2001b). Here we describe experiments designed to monitor long-term remodeling of the PSD in living neurons. To this end, we prepared primary hippocampal neurons from transgenic embryos expressing GFP-tagged PSD-Zip45 or PSD95. This method allowed us to visualize the PSD structure at $24 \mathrm{hr}$ intervals and thus permitted the detailed analysis of long-term synapse remodeling.

\section{Materials and Methods}

Generation of transgenic mouse lines carrying the GFP-PSD-Zip45 or PSD$95-G F P$ sequences together with the $\beta$-actin promoter. The chicken $\beta$-actin promoter was obtained from the plasmid vector pBactCAT9. The promoter region was ligated with the GFP-PSD-Zip45 or PSD-95-GFP coding region and the polyadenylation sequences of the pEGFP-N1 vector (Clontech, Palo Alto, CA) to generate pAct-GFP-PSD-Zip45 and pActPSD-95-GFP plasmids. Fertilized eggs from B6C3F1 mice received microinjections of the transgene. Integration of the transgene was detected by genomic PCR of the tail DNA.

Immunohistochemistry of the transgenic mouse. Animals were killed under sodium pentobarbital anesthesia and perfused transcardially with $2 \%$ paraformaldehyde in PBS. Brains were excised and cut with a vibratome to obtain $50 \mu \mathrm{m}$ sections. Sections were blocked with 5\% normal goat serum (NGS) and incubated with rabbit polyclonal antisynapsin I antibody (Chemicon, Temecula, CA) for GFP-PSD-Zip45 transgenic mice or with the mixture of rabbit polyclonal anti-GFP (Molecular Probes, Eugene, OR) and mouse monoclonal anti-synaptophysin (Roche Applied Science, Indianapolis, IN) for PSD-95-GFP transgenic mice. Primary antibodies were visualized with goat-anti rabbit IgG conjugated to Cy3 (Jackson ImmunoResearch, West Grove, PA) and goatanti mouse IgG conjugated to Alexa 488 (Molecular Probes). The samples were imaged with a Fluoview confocal laser-scanning microscope (Olympus Optical, Melville, NY).

Hippocampal cultures from transgenic embryos. Male mice heterozygous for the GFP-PSD-Zip45 or PSD-95-GFP transgenes were mated with wild-type females, and the embryos at embryonic day 17 (E17) were harvested and examined for the expression of transgenes under a fluorescence dissecting microscope. Hippocampal cultures from 17-d-old embryonic mice were prepared as described previously with slight modifications (Okabe et al., 1999, 2001a). The hippocampi from transgenic and wild-type embryos were mechanically dissociated in separate tubes without treatment with trypsin and DNase. Cells derived from transgenic embryos and those from wild-type embryos were mixed at a ratio of 1:9 and grown on glass coverslips attached to the bottom of dishes with holes of $10 \mathrm{~mm}$ diameter. Neurons were treated with $100 \mu \mathrm{M}$ Sp-cyclic adenosine 3',5'-monophosphorothioate (Sp-cAMPS; Biomol, Plymouth Meeting, PA). Sp-cAMPS application was started at $11 \mathrm{~d}$ in culture with renewal every $3 \mathrm{~d}$.

Immunocytochemistry. Polyclonal antibodies against guanylate kinaseassociated protein (GKAP) and cortactin-binding protein (CortBP) were produced by immunization of New Zealand White rabbits with glutathione S-transferase-fused GKAP and CortBP proteins (S. Usui and K. Sobue, unpublished data). Hippocampal neurons were fixed in $2 \%$ paraformaldehyde in PBS for 25 min or with methanol for $10 \mathrm{~min}$ at $-20^{\circ} \mathrm{C}$, blocked with $5 \%$ NGS, and incubated with mouse monoclonal anti-PSDZip45 (Tadokoro et al., 1999), rabbit polyclonal anti-GKAP, rabbit polyclonal anti-CortBP, mouse monoclonal anti-PSD-95 (Affinity Bioreagents, Golden, CO), rabbit polyclonal anti-GluR1 (Chemicon), rabbit polyclonal anti-NMDA receptor 1 (NR1; Chemicon), or rabbit polyclonal anti-synapsin I (Chemicon). Primary antibodies were visualized with goat anti-mouse or anti-rabbit IgG conjugated to Cy3 (Jackson ImmunoResearch).

Adenovirus infection. Generation and characterization of recombinant adenoviruses expressing PSD-95-yellow fluorescent protein (YFP) and synaptophysin-cyan fluorescent protein (CFP) were described previously (Kanegae et al., 1994, 1995; Miyake et al., 1996; Okabe et al., 2001a). Day 14 hippocampal cultures were exposed for $60 \mathrm{~min}$ to viruses at a multiplicity of infection of 100 . Cells were then washed, reincubated in the previously removed media, and after $48 \mathrm{hr}$ assayed by fluorescence microscopy.

Microscopy. For time-lapse imaging, live cells were mounted in a chamber at $37^{\circ} \mathrm{C}$ with a continuous flow of humidified $\mathrm{CO}_{2}$ to maintain the $\mathrm{pH}$ of the medium. Images were obtained on a Fluoview confocal laser-scanning microscope (Olympus Optical). A $60 \times$ water immersion lens was used, and images were collected at an additional electronic zoom factor of $3 \times$. Multiple optical sections ( $12-15$ sections and $z$-spacing of $0.3-0.4 \mu \mathrm{m}$ ) were collected, and these images were recombined using a maximum-brightness operation. Illumination by the $488 \mathrm{~nm}$ line of an argon ion laser was attenuated to $0.5-5 \%$ to reduce phototoxicity. We performed repetitive imaging of the same cell by using $x-y$ coordinates read from the custom stage micrometer.

Data analysis. Maximal intensity projection images were prepared for each image stack, and these projection images were used for the quantitative analysis. For a given time-lapse image series, all projection images were processed identically for cluster extraction and for quantitative analysis of fluorescence. Automatic processing of fluorescence images using Metamorph software (Universal Imaging, West Chester, PA) enabled us to determine the position, area, and intensity of fluorescent clusters. Briefly, projection images were processed with a low-pass filter, and the resulting images were subtracted from the original images. Images were then converted to binary images using intensity thresholding. These binary images were subjected to erosion and dilation procedures to remove single-pixel noise. The numbers of fluorescent clusters were counted using the integrated morphometry analysis function of the Metamorph software. To quantify the fluorescence intensity of clusters, the binary images were used to specify the cluster domains. The total fluorescence intensity and the average fluorescence intensity in each domain were measured. For PSD-95-GFP clusters, automatic thresholding of the images could not remove the background fluorescence from the dendritic shafts, mainly because of the low expression level of the PSD95-GFP. By comparing the original fluorescence images and the binary images, the regions containing the background of the dendritic shafts were manually set in the binary images and were excluded from the integrated morphometry analysis.

The correlation coefficient between two dendritic segments, $j$ and $k$, was calculated as:

$$
r_{\mathrm{jk}}=\frac{\sum_{\mathrm{i}=1}^{N}\left\{\left(C_{\mathrm{ji}}-\bar{C}_{\mathrm{j}}\right)\left(C_{\mathrm{ki}}-\bar{C}_{\mathrm{k}}\right)\right\}}{\sqrt{\sum_{\mathrm{i}=1}^{N}\left(C_{\mathrm{ji}}-\bar{C}_{\mathrm{j}}\right)^{2} \sum_{\mathrm{i}=1}^{N}\left(C_{\mathrm{ki}}-\bar{C}_{\mathrm{k}}\right)^{2}}},
$$

where $C_{\mathrm{ji}}$ is the number of GFP-PSD-Zip45 clusters on day $\mathrm{i}$ in a dendritic segment, $\mathrm{j}$, and $N$ is the total number of observations.

The cluster elimination index was calculated as:

$$
I=\frac{\left(\sum_{\mathrm{i}-1}^{N-1}\left|C_{\mathrm{i}+1}-C_{\mathrm{N}}\right|\right)-\left(C_{\mathrm{N}}-C_{1}\right)}{2 \sum_{i=1}^{N} C_{\mathrm{i}}},
$$

where $C_{\mathrm{i}}$ is the number of GFP-PSD-Zip45 clusters on day $\mathrm{i}$ in a dendritic segment, and $N$ is the total number of observations. The numerator 

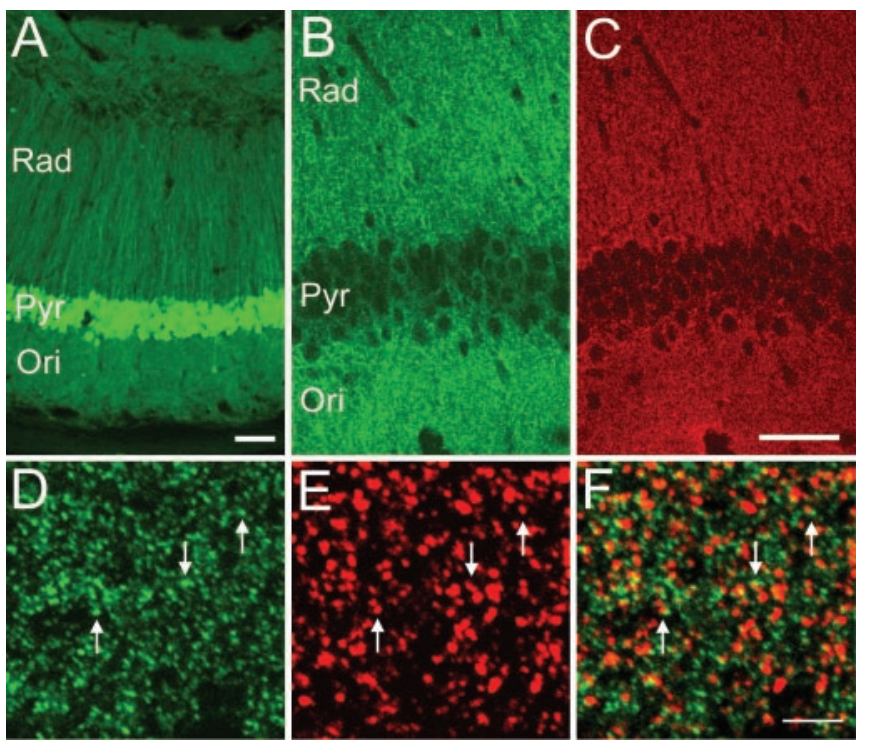

Figure 1. Expression of the GFP-PSD-Zip45 transgene in the hippocampus. A, Laser confocal microscopy of the CA1 region from $\beta$-actin promoter GFP transgenic mice revealed strong GFP fluorescence in the pyramidal cell layer (Pyr). Dendrites of CA1 pyramidal neurons also showed GFP fluorescence in the stratum radiatum (Rad) and stratum oriens (Ori). B, C, Laser confocal microscopy of the CA1 region from the $\beta$-actin promoter GFP-PSD-Zip45 transgenic mice revealed GFP fluorescence ( $B$, green) in both the stratum radiatum $(R a d)$ and the stratum oriens (Ori). The distribution of GFP-PSD-Zip45 was similar to that of synapsin I immunoreactivity (C, red). $D-F$, Higher-magnification view of the stratum radiatum of the $\beta$-actin promoter GFPPSD-Zip45 transgenic mice. GFP clusters ( $D$, arrows) were in close apposition to the clusters of synapsin I (E, arrows). F, Superposition of $D$ and $E$. Scale bars: $A-C, 50 \mu \mathrm{m} ; D-F, 5 \mu \mathrm{m}$.

corresponds to the cumulative number of eliminated clusters during the observation period. To calculate the proportion of dendritic domains with reappearance of PSD-Zip45 clusters, we first generated binary image stacks of GFP-PSD-Zip 45 clusters from 13 to $18 \mathrm{~d}$ in culture and selected two types of the circular dendritic regions $(2.0 \mu \mathrm{m}$ in diameter). The first dendritic regions were the domains positive with GFP-PSD-Zip45 clusters that disappeared in the second image. The second dendritic regions were the domains negative with GFP-PSD-Zip45 clusters in both the first and second images. After selecting the same number of dendritic regions with these two categories, the image stacks were analyzed to determine whether selected regions were positive with new GFP-PSD-Zip45 clusters from the third to sixth images in the stack.

\section{Results}

Preparation of hippocampal culture from GFP-PSD-Zip45 and PSD-95-GFP transgenic mice

Histological examination of transgenic mice expressing GFP under the control of the chicken $\beta$-actin promoter revealed widespread expression of GFP in CNS neurons. In the hippocampus, strong GFP fluorescence was observed in the cell bodies of CA1 pyramidal neurons (Fig. $1 A$ ). In transgenic mice expressing GFPPSD-Zip45 under the control of the chicken $\beta$-actin promoter, the GFP fluorescence within the hippocampal CA1 region was restricted to the stratum radiatum and stratum oriens, with little fluorescence in the cell bodies of pyramidal cells (Fig. 1B,C). Clusters of GFP-PSD-Zip45 were associated with the clusters of synapsin I in the stratum radiatum (Fig. $1 D-F$ ). This distribution is consistent with the postsynaptic localization of PSD-Zip45 in CA1 pyramidal neurons. Three independent lines of transgenic mice mated normally and showed normal growth and body weight. No gross abnormalities of the brain structure were observed.

To develop a culture system that would allow us to observe long-term remodeling of the PSD structure, we prepared mixed cultures of GFP-PSD-Zip45-expressing cells and wild-type cells. To do this, male mice heterozygous for the GFP-PSD-Zip45 transgene were mated with wild-type females, and the embryos at E17 were harvested and examined for the expression of GFPPSD-Zip45 under a fluorescence dissecting microscope. At this stage, the fluorescence in the CNS was prominent in the spinal cord and the midbrain region. The hippocampi from transgenic and wild-type embryos were dissociated separately, and cells derived from transgenic embryos and those from wild-type embryos were mixed at a ratio of 1:9 and grown on glass coverslips. In these experimental conditions, GFP-PSD-Zip45-expressing cells were separated from each other, and the identification of single dendrites was possible (Fig. $2 A-E$ ).

We generated multiple transgenic lines of PSD-95-GFP under the control of the chicken $\beta$-actin promoter. Expression of GFPtagged proteins in these lines was generally lower than the expression in GFP-PSD-Zip45 transgenic lines. We selected a single line that showed the highest expression of PSD-95-GFP and examined the distribution of PSD-95-GFP by immunohistochemistry using anti-GFP antibody. Distribution of PSD-95-GFP within the hippocampal CA1 region was restricted to the stratum radiatum and stratum oriens (Fig. $3 A, B$ ), and the anti-GFP immunopositive puncta were associated with the anti-synaptophysin immunopositive presynaptic structures (Fig. 3C-E). The culture protocol for GFP-PSD-Zip45 mice was also applied to the embryos of PSD-95-GFP transgenic mice, and fluorescence signals from PSD-95-GFP-expressing pyramidal neurons could be visualized under the illumination condition of 5- to 10-fold higher laser intensity than that of GFP-PSD-Zip45 (Fig. 3F, G).

Within 1 week after plating, the majority of cells expressing GFPPSD-Zip45 and PSD-95-GFP showed weak diffuse fluorescence in dendrites and cell bodies. Fluorescent clusters started to appear in dendrites 7-12 d after plating. Therefore, we focused on the development of the PSD structure in neurons at 11-22 d in culture. Our previous studies on developing neurons in culture prepared using a similar protocol indicated substantial remodeling of the dendritic structure and the clustering of PSD molecules at the same developmental stage (Okabe et al., 2001a).

\section{Characterization of GFP-PSD-Zip45- and \\ PSD-95-GFP-expressing neurons}

In this study, we used the combination of GFP-tagged PSD molecules and transgene-mediated reporter expression to monitor the long-term remodeling process of the PSD structure. In this context, it is important to see whether fluorescent clusters faithfully represent the localization of the PSD structure in cultured hippocampal neurons. To this end, we fixed the culture preparations from GFP-PSD-Zip45 and PSD-95-GFP transgenic embryos at $11 \mathrm{~d}$ in culture and stained them with antibodies against six postsynaptic proteins: AMPA receptor subunit GluR1 (Fig. $2 D$ ), NMDA receptor subunit NR1, PSD-95 (Figs. 2A, 3F), PSD-Zip45 (Fig. 3G), CortBP (Fig. 2B), and GKAP (Fig. 2C).

We first performed quantitative analysis of colocalization of GFP-PSD-Zip45 clusters with clusters of other postsynaptic proteins. The results indicate that $84 \pm 4.1,91 \pm 2.5$, and $94 \pm 2.5 \%$ of PSD-Zip45 clusters colocalized with the clusters of PSD-95, GKAP, and CortBP, respectively (Fig. $2 F$ ). The level of fractional match was reasonably high, because the fractions of native PSD-95 clusters that colocalized with either CortBP or GKAP clusters were $85 \pm 3.1$ and $91 \pm 2.4 \%$, respectively. We also analyzed colocalization of PSD-95-GFP clusters with that of other PSD proteins at $11 \mathrm{~d}$ in culture. The results indicated that 

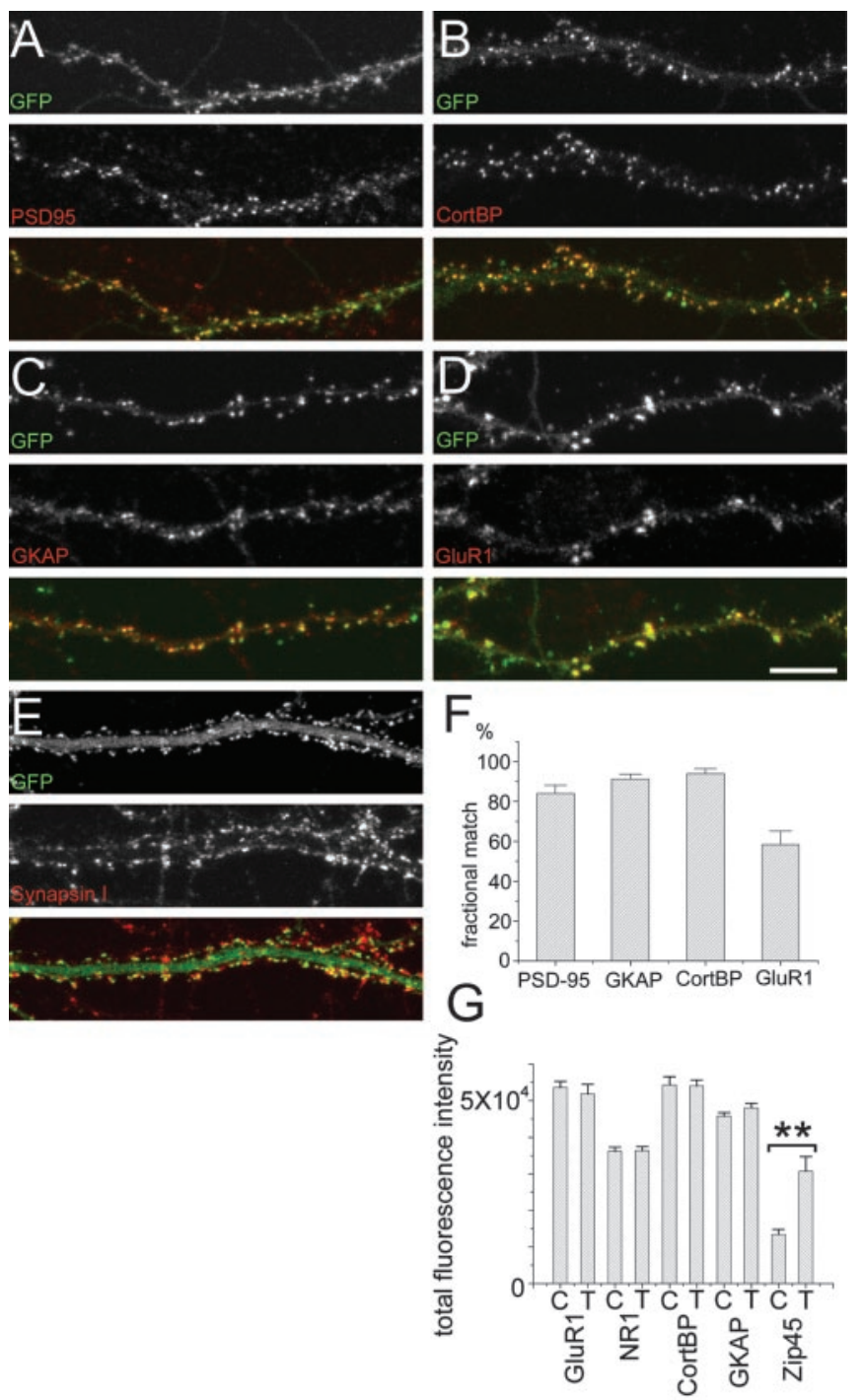

Figure 2. Localization of GFP-PSD-Zip45 in cultured hippocampal neurons isolated from the GFP-PSD-Zip45 transgenic mice. A-E, Colocalization of GFP-PSD-Zip45 with PSD-95 ( $A$ ), CortBP $(B), \operatorname{GKAP}(C)$, GluR1 (D), and synapsin I (E). In the merged color images, the green channel shows the GFP-PSD-Zip45 distribution, and the red channel shows the immunoreactivity of synaptic markers. F, Fractional match of GFP-PSD-Zip45 clusters with PSD-95, GKAP, CortBP, and GluR1 immunoreactivities in the postsynaptic dendrites. G, Effects of GFP-PSD-Zip45 overexpression by the transgene on the assembly of PSD molecules. Average fluorescence intensities of GluR1, NR1, CortBP, and GKAP clusters were not significantly different between the transgenic mice-derived neurons and the wild-type neurons. Average fluorescence intensity of PSDZip45 clusters immunostained with anti-PSD-Zip45 antibody showed 130\% overexpression of PSD-Zip45 in the transgenic mice-derived neurons. This difference was statistically significant $\left.{ }^{* *} p<0.01\right)$. Scale bar, $10 \mu \mathrm{m}$.

$86 \pm 6.3,90 \pm 3.5$, and $92 \pm 6.0 \%$ of PSD-95-GFP clusters colocalized with the PSD-Zip45, GKAP, and CortBP clusters, respectively. To determine whether the degree of colocalization increases with the maturation of neurons in culture, we determined the percent colocalization between PSD-Zip45 and PSD-95 at $22 \mathrm{~d}$ in culture. The fractions of GFP-PSD-Zip45 clusters and PSD-95-GFP clusters that colocalized with native PSD-95 and PSD-Zip45 were $90 \pm 4.6$ and $93 \pm 3.0 \%$, respectively. Thus there was a slight increase of the colocalization as the maturation of neurons proceeded in culture.

The AMPA receptor subunit GluR1 is also concentrated at postsynaptic sites, but its distribution is also regulated by synaptic activity. Our immunofluorescence study revealed that $58 \pm 6.7 \%$ of PSD-Zip45 clusters colocalized with the clusters of GluR1 (Fig. $2 D, F)$. This lower factional match between AMPA receptor clusters and scaffolding proteins in the PSD was consistent with results of previous studies that showed activity-dependent accumulation of AMPA receptors at excitatory synapses (Liao et al., 1999). Immunofluorescence analysis of cultured neurons from transgenic mice also revealed a close association of synapsin I-positive presynaptic structures with the PSD-Zip45 clusters (Fig. 2E).

Recent studies using transfection techniques of primary neurons showed that overexpression of either PSD-95 (El-Husseini et al., 2000), Shank (an isoform of CortBP proteins; Sala et al., 2001), or spine-associated Rap-specific GTPase activating protein (Pak et al., 2001) leads to the enhancement of synaptic transmission, morphological maturation of the postsynaptic structures, or both. We measured the amount of synaptic PSDZip45 in neurons derived from both transgenic and wild-type embryos. The anti-PSD-Zip45 staining levels in puncta of GFPPSD-Zip45-expressing neurons were $130 \%$ higher on average than in wild-type neurons (Fig. 2G). This level of PSD-Zip45 overexpression was comparable with that obtained by adenovirus-mediated gene transfer and was shown to have negligible effects on the distribution of other PSD proteins (Okabe et al., 2001b). To further confirm that transgenic overexpression of PSD-Zip45 did not alter synaptic characteristics, we measured the amount of synaptic GluR1, NR1, CortBP, and GKAP in neurons derived from both transgenic and wild-type embryos. The staining levels of GluR1, NR1, CortBP, and GKAP did not show any statistical differences between GFP-PSD-Zip45-expressing cells and wild-type cells (Fig. 2G).

Next we determined the level of overexpression of PSD-95 in PSD-95-GFP-expressing neurons. The amount of synaptic PSD-95 immunoreactivity was only $18 \%$ higher than in wild-type neurons. This level of overexpression was 25 -fold less than the expression level known to promote the synaptic maturation (ElHusseini et al., 2000, 2002). In addition, we did not observe an increase of PSD-Zip45 immunoreactivity in neurons expressing PSD-95-GFP (Fig. 3H). Taken together, it is less likely that the transgene-mediated expression of GFP-tagged PSD molecules induced maturation of excitatory synapses.

\section{Visualization of long-term remodeling of the \\ PSD-Zip45 clusters}

A stable and higher expression level of GFP-PSD-Zip45 was advantageous to perform repetitive imaging of multiple dendrites with a lower illumination level. Therefore, we first focused on the GFP-PSD-Zip45-expressing neurons and obtained images of multiple primary or secondary dendrites from three or four cells on single coverslips attached to the culture chambers at $24 \mathrm{hr}$ intervals over 11-22 d in culture. Dendrites at 11-13 d after plating frequently possessed numerous filopodia-like extensions that were negative for fluorescent clusters (Fig. 4A,B). Repeated observation of the same dendritic segments illustrated a disappearance of thin filopodia and an increase of spines containing clusters of PSD-Zip45. This result is consistent with results of our previous analysis of developing hippocampal neurons fixed and stained with the lipophilic dye DiI (Okabe et al., 2001a). To confirm that newly generated fluorescent clusters corresponded to authentic synaptic sites, we fixed the cells after time-lapse imaging and immunostained them with antibodies against CortBP (Fig. 4C) and GKAP (Fig. 4D). More than $90 \%$ of the newly 
formed clusters colocalized with CortBP and GKAP immunoreactivity (95\% for CortBP and $91 \%$ for GKAP). This result indicates that most newly generated PSDZip45 clusters reflect the clustering sites of multiple PSD molecules.

We next performed quantitative analysis of the GFP-PSD-Zip45 clusters. Application of automated processing of digitized images successfully extracted the positions, sizes, and fluorescence intensities of PSD-Zip45 clusters (Fig. 5A). We measured the number of GFP-PSD-Zip45 clusters from 64 independent time-lapse sequences (images were obtained from 17 cells in five independent culture preparations). On average, PSD-Zip45 clusters increased from $0.32 \pm 0.04 / \mu \mathrm{m}$ at $11 \mathrm{~d}$ in culture to $0.729 \pm 0.09 / \mu \mathrm{m}$ at $17 \mathrm{~d}$ in culture (Fig. $4 E$ ). The calculated rate of cluster increase $\left(0.068 \cdot \mu \mathrm{m}^{-1} \cdot \mathrm{d}^{-1}\right)$ was comparable with our previous data on the development of PSD-95 clusters $\left(0.048 \cdot \mu \mathrm{m}^{-1} \cdot \mathrm{d}^{-1}\right.$; Okabe et al., 1999). The steady increase of GFP-PSD-Zip45 clusters suggests that repeated imaging of the same dendritic fields does not severely impair normal synaptogenesis.

Temporal profiles of GFP-PSD-Zip45 cluster density in individual dendrites showed a variety of patterns. Some dendrites showed monotonous increases in clusters, whereas others showed complex patterns, including short decremental stages. To classify the pattern of cluster increase, we calculated linear regression between days in culture and the normalized number of fluorescent clusters. When the regression lines showed positive slopes with a significance level of $p<0.05$, image sequences were classified as dendrites with monotonous patterns of cluster increase. Image sequences with a significance level of $p>0.05$ were classified as dendrites with complex patterns of cluster remodeling. Image sequences with a negative slope of the regression line showed a significance level of $p>0.05$. There was reasonably good correspondence between image sequences classified by this method and by eye. We identified 27 image sequences that showed a monotonous increase of clusters in 11 cells and 37 image sequences that showed complex patterns of cluster remodeling in 14 cells. In the following sections, we first describe the analysis of dendrites with monotonous cluster increases to illustrate the basic mechanisms of PSD-Zip45 cluster formation. Subsequently, an analysis of the synchronization of cluster addition and elimination in cells showing complex patterns of PSD-Zip45 cluster remodeling is described.

\section{Analysis of dendrites showing a monotonous increase in the PSD-Zip45 clusters}

We first focused on dendritic segments that showed monotonous increases in the GFP-PSD-Zip45 clusters to reveal the basic mechanisms of cluster assembly (Fig. 5A). Quantitative analysis of dendrites showing monotonous increases in clusters revealed that the mean fluorescence intensities of individual clusters were stable over time (Fig. 5B). Although there was a slight decrease in the total fluorescence in individual clusters (Fig. 5C), this differ- ence was not statistically significant (one-way ANOVA, $F=0.72$; $p>0.05)$. In dendritic shafts, the level of diffuse fluorescence signals was stable (Fig. 5B). This fraction is likely to represent a cytosolic pool of GFP-PSD-Zip45 molecules. In contrast, the sum of the intensity values in all clusters increased 1.8-fold during the observation period (Fig. 5D). These results indicate two properties of PSD-Zip45 cluster formation. First, the increase of PSDZip45 clusters can be explained by the net increase of the total amount of PSD-Zip45 molecules in dendrites. In other words, it is not attributable to the change in the local balance between the assembled and dissociated PSD-Zip45 molecules. Second, increases in the cluster density are related to net increases in the cluster number with little change in the size of individual clusters.

\section{Rapid increase of PSD-Zip45 clusters in newly generated dendritic segments}

Formation of new clusters and their remodeling occurred simultaneously with structural changes of dendrites. We identified 21 image sequences that showed formation of dendritic segments of $>20 \mu \mathrm{m}$ from 64 time-lapse sequences. These sprouting dendrites rapidly acquired new PSD clusters, and their density caught up with the level of parental dendrites within a few days (Fig. $6 \mathrm{~A}$ ). The patterns of cluster increase in all sprouting dendrites were classified as monotonous. The increase of PSD-Zip45 clusters in the newly generated dendritic segments was significantly faster than that in the parental dendrites (Fig. 6B,C). These results suggest the presence of regulatory mechanisms that enhance the formation of PSD structures in 

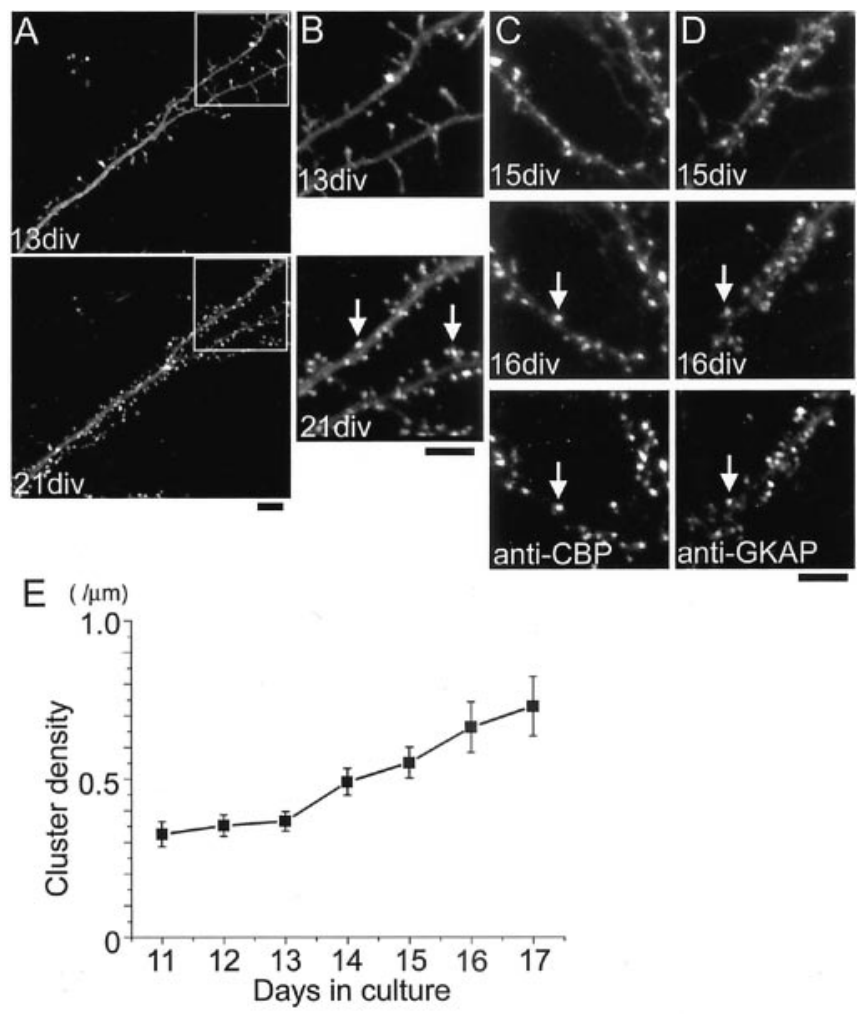

Figure 4. Long-term remodeling of GFP-PSD-Zip45 clusters in cultured hippocampal neurons. $A, B$, Remodeling of the same dendrite from 13 to $21 \mathrm{~d}$ in culture (div). $B$, Highermagnification views of the regions enclosed by the white boxes in $A$. Thin filopodia disappeared, and short spines with GFP clusters within their heads were generated (arrows). C, D, Colocalization of newly formed GFP-PSD-Zip45 clusters with other PSD molecules. Newly generated GFP clusters (arrows) between 15 and $16 \mathrm{~d}$ in culture were immunoreactive with anti-CortBP antibody (anti-CBP) and anti-GKAP antibody (anti-GKAP). E, Steady increase of the average density of GFP-PSD-Zip45 clusters in culture. Scale bars, $5 \mu \mathrm{m}$.

sprouting dendrites and thereby maintain the uniformity of PSDZip45 cluster density within individual neurons.

Synchronized addition and elimination of PSD-Zip45 clusters We next analyzed dendritic segments that showed complex patterns of cluster increase. Here we again extracted the positions, sizes, and fluorescence intensities of GFP-PSD-Zip45 clusters by automated processing of digital images. In the image sequence shown in Figure 7A, GFP-PSD-Zip45 clusters decreased gradually until $17 \mathrm{~d}$ in culture and rapidly increased in number in a subsequent $24 \mathrm{hr}$ period. A similar rapid increase of the cluster number took place in multiple time-lapse sequences ( 8 cases in 37 image sequences). Quantitative analysis revealed that total fluorescence intensities derived from clusters changed according to the number of clusters in the segment, but the diffuse fluorescence signal within the dendritic shaft remained constant (Fig. $7 B$ ). This result indicates that the assembly and disassembly of PSD-Zip45 clusters are not dependent on the local concentration of cytosolic PSD-Zip45 molecules. To determine whether new clusters show a tendency to reappear on the same dendritic positions, we calculated the proportion of cluster reappearance to the sites of previous cluster disappearance. The calculated proportion $(21.1 \%$, data from seven dendrites of the complex remodeling pattern) was not higher than the value obtained from the dendritic domains without previous clusters $(23.7 \%$, data from seven dendrites of the complex remodeling pattern). This result

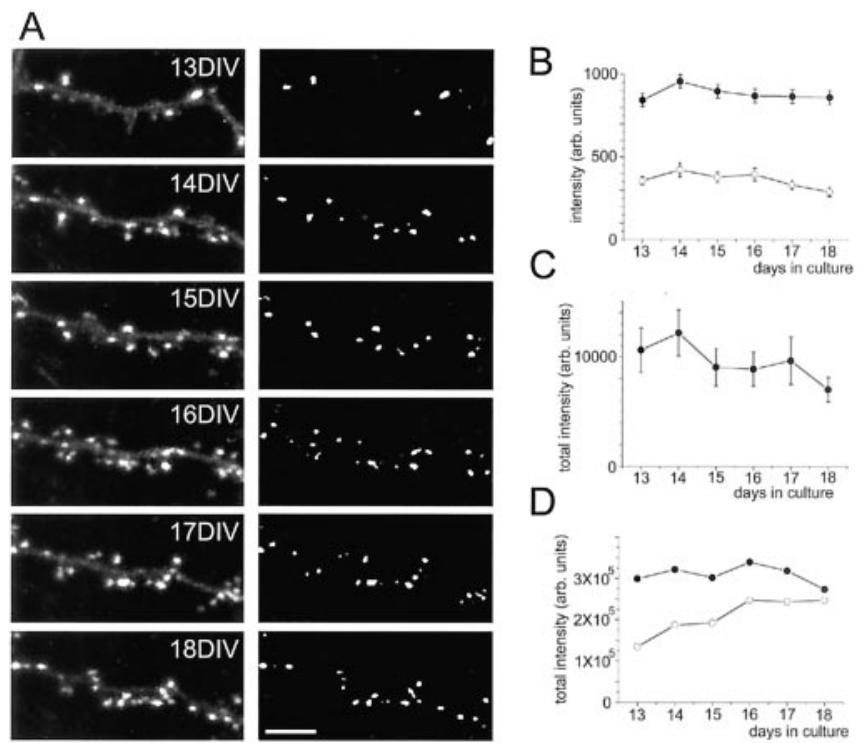

Figure 5. Dendritic segments showing monotonous increase of the GFP-PSD-Zip45 clusters. $A$, Time-lapse images of the dendritic segment showing monotonous increase of the clusters. The right panels show the binary images of clusters extracted from the original fluorescence images on the left. $B$, Changes in the average intensity values of the GFP-PSD-Zip45 clusters ( filled circles) and the dendritic shaft (open circles).C, Changes in the sum of the intensity values within individual GFP-PSD-Zip45 clusters. The differences in the total fluorescence per cluster were not statistically significant. D, Changes in the sum of the intensity value in all clusters (open circles) and within the shaft region (filled circles). There was a 1.8-fold increase of the total cluster intensity, whereas that within the dendritic shaft region remained stable. DIV, Days in culture. Scale bar, $5 \mu \mathrm{m}$.

indicates that PSD-Zip45 clusters do not disappear and reappear on the same position of the dendrites.

In image sequences with complex patterns of cluster increases, the majority of dendritic segments within a single cell appeared to increase or decrease the number of fluorescent clusters synchronously. If the developmental profiles of PSD-Zip45 clusters are regulated on a single-cell level, the patterns should be correlated within a single neuron and should be variable between different neurons. To test this possibility, the correlation in cluster development of GFP-PSD-Zip45 was compared among dendrites of the same cells and among dendrites originating from different cells (Fig. $8 A$ ). In this experiment, cells 1 and 3 showed a typical complex pattern of cluster remodeling (Fig. $8 \mathrm{~B}$ ). The patterns of cluster remodeling appeared to be similar among dendritic segments of the same cell but were distinct among dendritic segments originating from different cells. The average correlation coefficients between dendritic segments from the same cells and from different cells were calculated (Fig. 8C). Analysis of five sets of culture preparations revealed that the correlation within the same cell was significantly higher than that between different cells (Fig. 8D). To avoid the possible influence of local activity to the pattern of cluster remodeling, we selected 10 pairs of neurons located in the same vicinity (their cell bodies located within $400 \mu \mathrm{m}$ ) and performed the same analysis. Again the correlation within the same cell $(0.70 \pm 0.085)$ was significantly higher than that between different cells $(0.27 \pm 0.070 ; p<$ 0.01 ). Thus, even pairs of adjacent neurons show distinct patterns of cluster remodeling. This result is consistent with the notion that the pattern of PSD-Zip45 cluster development is regulated on a single-cell level.

To determine whether the temporal patterns of cluster formation within dendritic segments are correlated with the spatial distribution of dendritic segments, we calculated correlation co- 

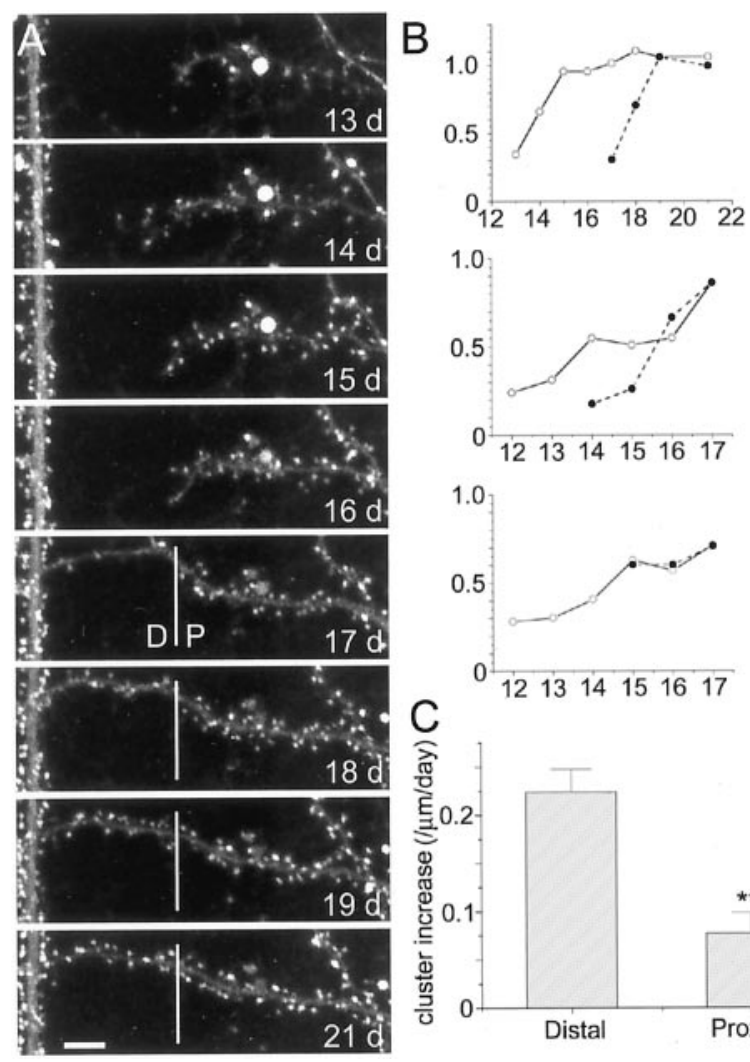

Figure 6. Rapid increase of GFP-PSD-Zip45 clusters in newly generated dendritic segments. $A$, Time-lapse images showing the initiation of dendritic sprouting at $17 \mathrm{~d}$ in culture. A newly generated dendrite in the distal portion $(D)$ formed fluorescent clusters rapidly, whereas the density of fluorescent clusters in the proximal dendritic segment $(P)$ was relatively stable. $B$, Changes in the densities of GFP-PSD-Zip45 clusters in the distal, newly formed dendritic segments (filled circles) and the proximal, preexisting dendritic segments (open circles). Measurements of three neurons from three independent culture preparations were presented. C, Rate of cluster density increase in the distal and proximal part of the sprouting dendrites. Data are derived from analysis of a total of nine neurons in five independent culture preparations $\left({ }^{* *} p<\right.$ 0.01). Scale bar, $5 \mu \mathrm{m}$.

efficients of the temporal patterns of cluster formation between pairs of dendritic segments $10 \mu \mathrm{m}$ in length (Fig. 9A,B). The correlation coefficient was 0.68 on average $(n=78)$, indicating that the pattern of cluster development is highly synchronized. Scatter plots of correlation coefficient versus distances between paired dendritic segments showed that there was no strong relationship between these two parameters. The same data set was analyzed in a different protocol. We grouped dendritic segments $10 \mu \mathrm{m}$ in length by their relationship to dendritic branching; namely, dendritic segments without any intervening branch points were grouped together. Again we plotted correlation coefficients of the pairs of dendritic segments within and between these groups (Fig. 9C). Analysis of four neurons that showed a complex pattern of cluster increase revealed that the correlation coefficients within the groups were not significantly greater than the values between groups (Fig. 9D). These results indicate that the patterns of PSD-Zip45 cluster development are regulated to maintain the uniformity of PSD density throughout the dendritic arborization within a single cell.

\section{Long-term remodeling of PSD-95 clusters}

To determine whether similar long-term remodeling took place in PSD-95 clusters, we obtained images of dendrites from PSD-95GFP-expressing cells at 24 -hr intervals over 11-18 d in culture. The
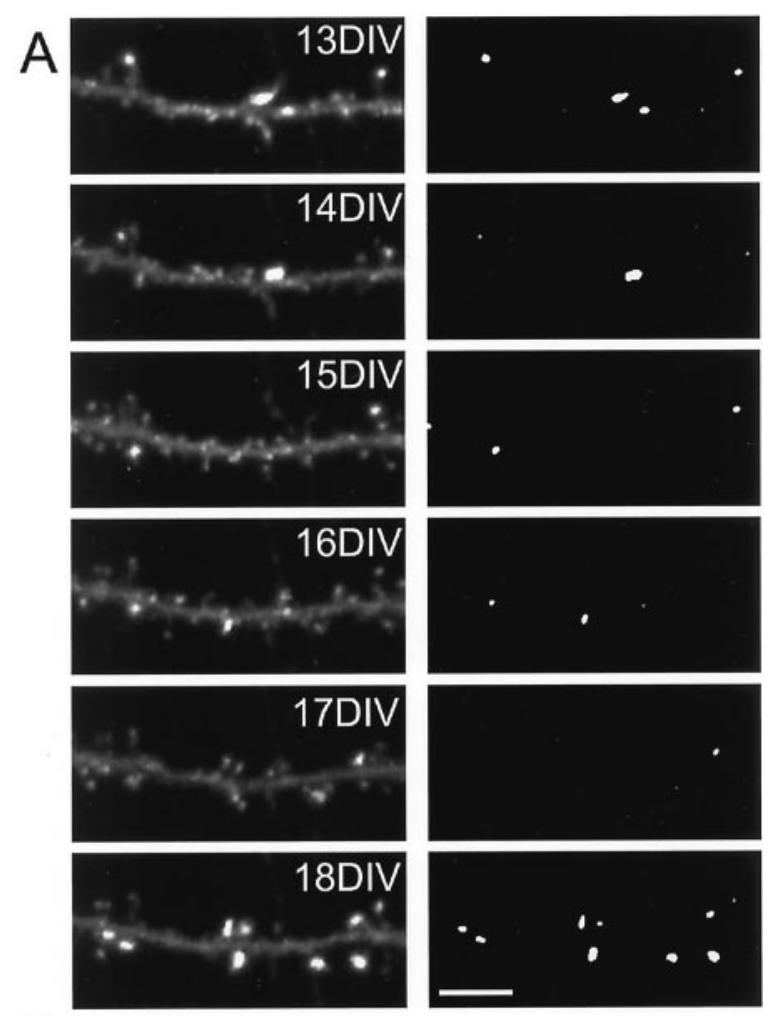

B

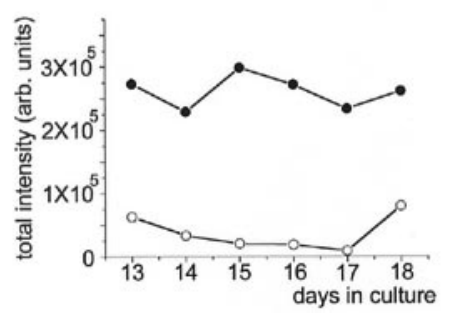

Figure 7. Dendritic segments showing a complex pattern of GFP-PSD-Zip45 cluster remodeling. $A$, Time-lapse images of the dendritic segment showing a complex pattern of cluster remodeling. The right panels show the binary images of clusters extracted from the original fluorescence images on the left. $B$, Changes in the total intensity values within clusters (open circles) and within the shaft region (filled circles). The total intensity values of the clusters showed a gradual decrease and a subsequent abrupt increase (open circles), whereas those within the dendritic shaft region were relatively stable (filled circles). DIV, Days in culture. Scale bar, $5 \mu \mathrm{m}$.

fluorescence intensity of PSD-95-GFP clusters was lower than that of PSD-Zip45-GFP clusters even in the imaging conditions of elevated laser intensity. To avoid phototoxic effects, we restricted the recording regions to two secondary dendrites of each neuron. In the image processing of PSD-95-GFP clusters, we have to include the step of manually eliminating the background fluorescence signals to determine the regions of fluorescent clusters (see Materials and Methods). Nevertheless, it was possible to identify PSD-95-GFP clusters (Fig. $10 A, B)$ and to determine the density of the clusters per unit length of the dendrites (Fig. 10C). Transient PSD-95 clusters were frequently observed (Fig. 10A,B, arrows), and a rapid increase of PSD-95 clusters within $24 \mathrm{hr}$ (Fig. 10 B, 16 DIV, 17 DIV), similar to the behavior of PSD-Zip45 clusters (Fig. 7A), was detected. We measured the number of PSD-95 clusters from 26 independent time-lapse sequences (images were obtained from 13 cells in four independent culture preparations). Temporal profiles of PSD-95-GFP cluster density can be classified into two categories: the monotonous pattern of cluster increase and the complex pattern of remodeling. We 
A
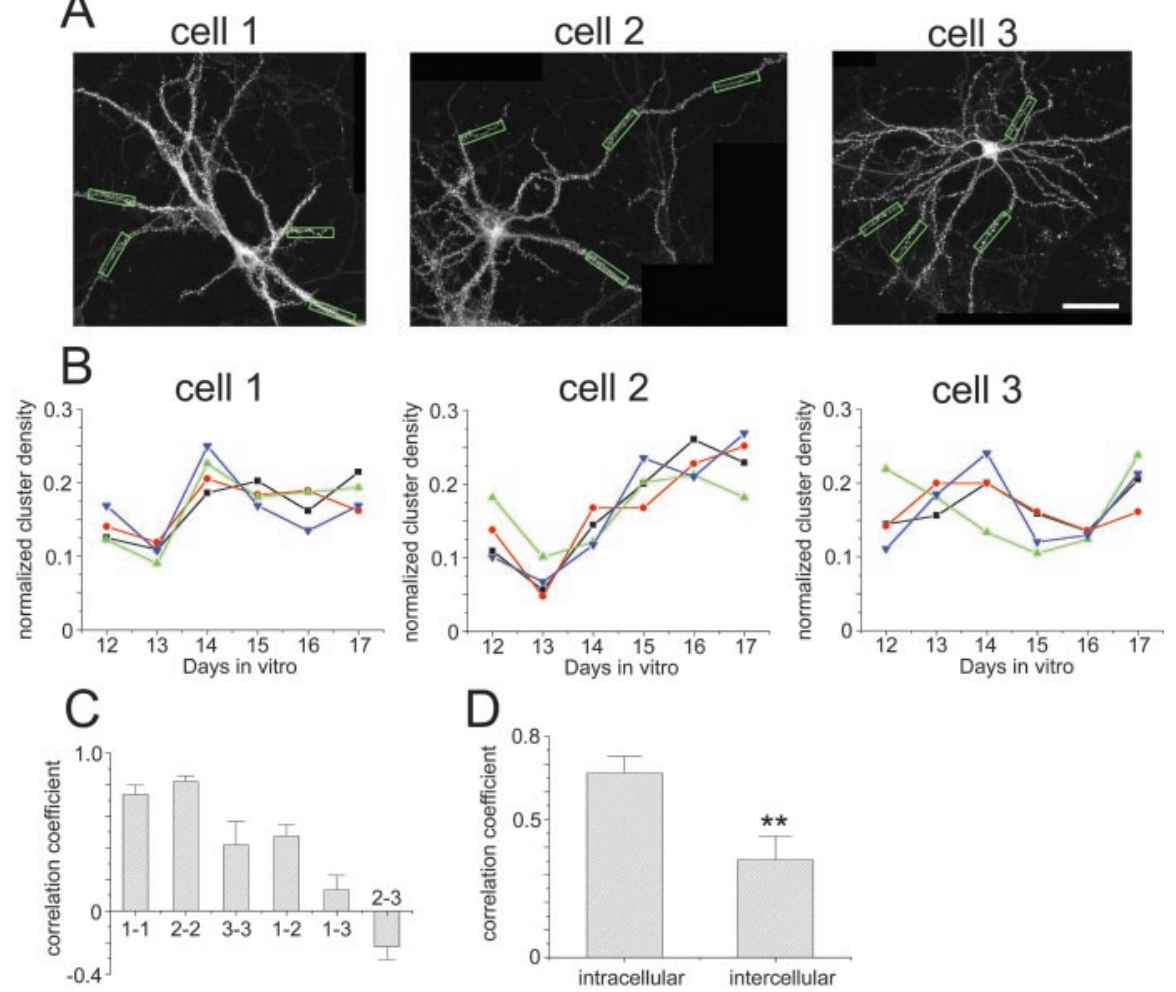

Figure 8. Correlation of the pattern of GFP-PSD-Zip45 cluster change within single neurons. A, Dendritic morphology of three hippocampal neurons expressing GFP-PSD-Zip45 in the same culture preparation. The densities of fluorescent clusters within the region enclosed by the green rectangles were measured. $B$, Patterns of the cluster density change in the four dendritic regions shown in $A$. The patterns between the dendrites of the same cell appeared to be similar, whereas the patterns between the different cells appeared to be distinct. C, Correlation coefficients calculated from the temporal patterns shown in $B$. There was a tendency that the average correlation coefficients between dendrites of the same cell $(1-1,2-2,3-3)$ were higher than those between different cells $(1-2,1-3,2-3)$. D, The average correlation coefficients between dendrites of the same cell were significantly higher that those between different cells. Data are derived from analysis of a total of 60 dendritic segments in five independent culture preparations $\left({ }^{* *} p<0.01\right)$. Scale bar, $50 \mu \mathrm{m}$.

identified 14 image sequences in the former category (Fig. 10A) and 12 in the latter category (Fig. $10 \mathrm{~B}$ ). This result is consistent with the multiple patterns of PSD-Zip45 remodeling described previously.

We next compared the correlation in cluster development of PSD-95-GFP among dendrites of the same cells and among those of different cells (Fig. 10C). The patterns of cluster remodeling appeared to be similar among dendritic segments of the same cells. This difference was statistically significant in the analysis of three sets of culture preparations (Fig. 10D). Thus, correlated remodeling of GFP clusters within cells was confirmed in the two distinct PSD molecules, PSD-Zip45 and PSD-95.

\section{Effects of activating cAMP-dependent signaling on PSD remodeling}

Generation and elimination of synapses and their molecular composition are regulated by neuronal activity and intracellular signaling mechanisms (Rao and Craig, 1997; Turrigiano et al., 1998; Liao et al., 1999; Murthy et al., 2001). Therefore, we next tested whether pharmacological manipulation of signaling mechanisms can influence the long-term remodeling of PSD-Zip45 clusters. We could not test the effects of blocking sodium channel activation or fast glutamatergic transmission because of the deteriorating effects of prolonged incubation with the sodium channel blocker TTX and the AMPA-type glutamate receptor blocker CNQX in our culture system. Phosphorylation is a candidate means for regulating synchronized remodeling of the PSD pro- teins. Previous studies showed involvement of the cAMP-dependent protein kinase (PKA) in synaptic potentiation (Kameyama et al., 1998; Lee et al., 2000) and recruitment of postsynaptic proteins (Crump et al., 2001; Gomez et al., 2002). Application of the membrane-permeable cAMP analog Sp-cAMPS induced suppression of the decremental stage in the temporal profiles of cluster change (Fig. $11 A, B)$. Prolonged incubation with SpcAMPS did not affect the increase of the average cluster density. We also observed a general increase of the temporal correlation between dendritic segments. This effect was mainly attributable to the less complex temporal profiles of cluster remodeling.

Distribution of PSD-Zip45 can be modulated by the neuronal activity (Okabe et al., 2001b), and the effect of SpcAMPS might be specific to the PSD molecules with dynamic properties similar to PSD-Zip45. We first analyzed acute effects of Sp-cAMPS application to the PSD-Zip45 dynamics. In contrast to the effects of NMDA receptor activation and membrane depolarization, application of Sp-cAMPS did not induce rapid redistribution of PSD-Zip45 (data not shown). Next we performed retrospective immunocytochemistry of GFP-PSD-Zip45expressing neurons with anti-PSD-95 antibody. If cAMP-dependent signaling is only related to the dissociation of PSDZip45 away from the core PSD structure and does not influence the remodeling of the entire postsynaptic structure, dendritic domains that lost GFP-PSD-Zip45 clusters should be still immunoreactive with anti-PSD-95 antibody. However, retrospective immunocytochemistry

revealed that $>90 \%$ of the dendritic domains that had lost GFP-PSD-Zip45 clusters were immunonegative with antiPSD-95 antibody (Fig. 11C,D). Furthermore, this correlation between PSD-Zip45 disappearance and the absence of PSD-95 immunoreactivity was not influenced by the treatment with Sp-cAMPS. Thus, it is less likely that Sp-cAMPS suppressed the dynamics of a specific subset of the PSD molecules.

\section{Small number of synaptic contacts generated by a single afferent fiber}

In culture conditions, the number of neurons projecting to a single pyramidal neuron should be much smaller than the number in the intact hippocampus. If a single neuron receives synaptic inputs from only a few afferent fibers, the synchronized pattern of PSD remodeling in a single cell can be explained by strong influences of these specific afferent fibers. To estimate the number of the PSDs associated with presynaptic structures originating from a single afferent, we infected wild-type hippocampal neurons in culture with two types of recombinant adenoviruses carrying either PSD-95-YFP cDNA or synaptophysin-CFP cDNA. By adjusting the titers of these two adenoviruses, it was possible to minimize the number of cells expressing both marker proteins. 
We selected neurons expressing PSD-95YFP with their dendritic fields isolated from other neurons and recorded the images of the entire dendritic field where a single axon expressing synaptophysin-CFP formed synaptic contacts (Fig. $12 A-C)$. On average, only $11.7 \%$ of total PSD-95 clusters were associated with synaptophysin-CFP clusters (Fig. 12D). Therefore, the contribution of a single axon to the total synaptic contacts on a single postsynaptic neuron was small, and the synchronized pattern of the PSD remodeling cannot be explained by the strong influence of a single afferent fiber.

\section{Discussion}

This study aimed to illustrate how individual PSD structures are assembled and eliminated in the stage of active synaptogenesis. Although the average density of GFP-tagged PSD clusters showed a steady increase from 11 to $17 \mathrm{~d}$ in culture, the temporal patterns of cluster density within individual dendritic segments were heterogeneous and complex. Comparison of the temporal profiles of cluster density among neurons showed synchronization of cluster addition and elimination within a single cell. This experimental evidence suggests an active and integrating role of the dendrite in the homeostasis of synapse density.

We previously performed repeated imaging of PSD-95 clusters expressed by adenovirus-mediated gene transfer in cultured hippocampal neurons and presented evidence that the rate of addition and elimination of PSD-95 clusters was much higher than the net increase of PSD-95 cluster density (Okabe et al., 1999). Although this type of study using transfection or virus-mediated gene transfer provided information on synapse remodeling in time scales from seconds to hours, prolonged observation of single neurons over days or weeks was difficult, mainly because of variable and unstable expression of GFP-tagged molecules. By using neurons derived from transgenic mice, we could achieve very stable expression of GFP-tagged PSD molecules in culture. This property enabled us to extract the number, location, and intensity of fluorescent clusters automatically and to perform detailed quantitative analyses. The pattern of the development of the PSD structure observed in this study was consistent with our previous studies using PSD95-GFP adenovirus (Okabe et al., 1999, 2001a); namely, there was a consistent and gradual increase in the average density of PSDs in culture. On the other hand, addition and elimination of individual PSD structures took place at a rate much higher than the net increase of PSDs.

One possible caveat to our present analysis is the validity of GFP-PSD-Zip45 fluorescence as a marker of the PSD structure. Our immunocytochemical analysis indicated extensive overlap of GFP-PSD-Zip45 clusters with other postsynaptic molecules, such as PSD-95, GKAP, and CortBP. This result supports the notion that PSD-Zip45 can be used as a probe to visualize the PSD dynamics. On the other hand, we previously compared the behavior of GFP-tagged PSD-95 and PSD-Zip45 molecules and presented evidence that a small number $(\sim 10 \%)$ of GFPPSD-Zip45 clusters change their fluorescence intensity rapidly (Okabe et al., 2001b). Therefore, it is possible that $10 \%$ of the GFP-PSD-Zip45 clusters are transient, and their turnover is not correlated with the behavior of other clusters. However, we showed that $>90 \%$ of newly generated GFP-PSD-Zip 45 clusters were immunopositive with other markers of the PSD, such as GKAP and CortBP. Furthermore, the degree of cluster remodeling during a period of $24 \mathrm{hr}$ was frequently more than a twofold increase or decrease. This dramatic change in cluster density cannot be explained merely by the small transient fraction of the PSD-Zip45 clusters. Finally, we showed that the behavior of PSD95, which does not show activity-dependent rapid recruitment (Okabe et al., 1999, 2001b), was similar to that of PSD-Zip45 in long-term visualization experiments described here. Taken together, it is less likely that the observed pattern of cluster addition and elimination was severely influenced by the transient PSDZip45 clusters. Although our data provided evidence of synchronous remodeling of PSDs containing PSD-95 and PSD-Zip45 molecules, it is still possible that other postsynaptic components, such as AMPA receptors and their cytoplasmic binding proteins, behave differentially (Fig. 2D). Even in mature hippocampal neurons, only $50-60 \%$ of NMDA receptor-containing synapses were positive with AMPA receptors (Liao et al., 1999). A large number of studies provided evidence of rapid redistribution of 


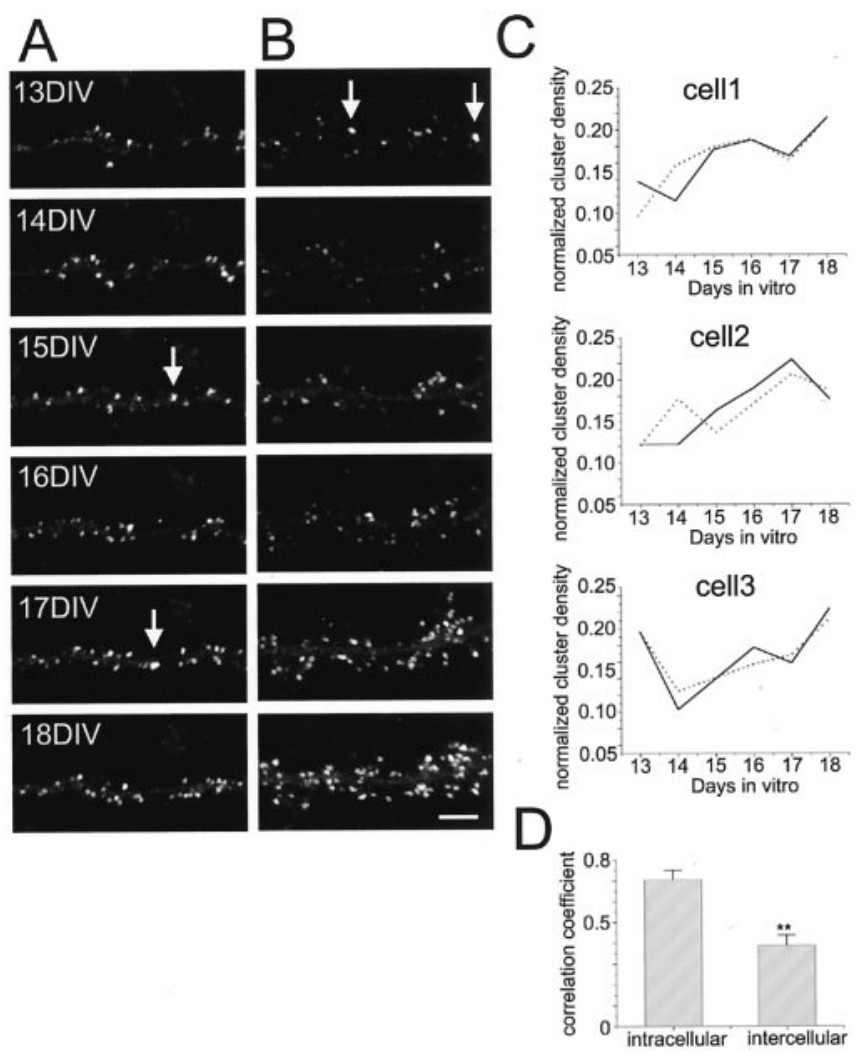

Figure 10. Long-term remodeling of PSD-95-GFP clusters in cultured hippocampal neurons. $A, B$, Remodeling of the same dendrites from 13 to $18 \mathrm{~d}$ in culture (DIV). Image sequences in $A$ show a dendritic segment with monotonous increase of the PSD-95 clusters. Although the density of the PSD-95-GFP clusters increased with time, disappearance of preexisting clusters was frequent ( $A$, arrows). A dendritic segment showing a complex pattern of PSD-95-GFP cluster remodeling $(B)$ was also observed. In this image sequence, large PSD-95-GFP clusters present in the first image (arrows) were disassembled $24 \mathrm{hr}$ later. Subsequently, rapid increase of the PSD-95-GFP clusters was observed at 17 and $18 \mathrm{~d}$ in culture. Scale bar $(A, B), 5 \mu \mathrm{m}$. $C$, Patterns of the cluster density change in two dendritic regions of three cells present in the same culture preparation. The patterns between the dendrites of the same cells appeared to be similar, whereas the patterns between the different cells were distinct. $D$, The average correlation coefficients between dendrites of the same cell were significantly higher than those between different cells. Data are derived from analysis of a total of 26 dendritic segments in four independent culture preparations $\left({ }^{* *} p<0.01\right)$.

AMPA receptors after stimulation (Lissin et al., 1999; Shi et al., 1999; Hayashi et al., 2000). Simultaneous long-term imaging experiments of AMPA receptors and PSD proteins, including PSD-95 and PSD-Zip45, will be necessary to clarify the relationship between rapid redistribution of AMPA receptors and longterm remodeling of PSD proteins described in this study.

Filopodia are abundant in immature dendrites and are subsequently replaced by stubby, mushroom-shaped spines. This morphological transition was proposed initially from electron microscopic analysis of the hippocampus in vivo (Schwartz et al., 1968; Harris et al., 1992), and supporting evidence in culture preparations has also accumulated (Ziv and Smith, 1996; Okabe et al., 2001a). However, the evidence was derived mainly from the comparison of cells in different culture preparations, and evidence that a single dendrite can change its morphology during development was lacking. Repeated observation of the same dendrite for $>1$ week enabled us to compare the structural remodeling of filopodia-rich dendrites to those covered by typical spines with clusters of PSD-Zip45 (Fig. 4A,B). Because our sampling frequency was low, we could not conclude that a single motile filop-
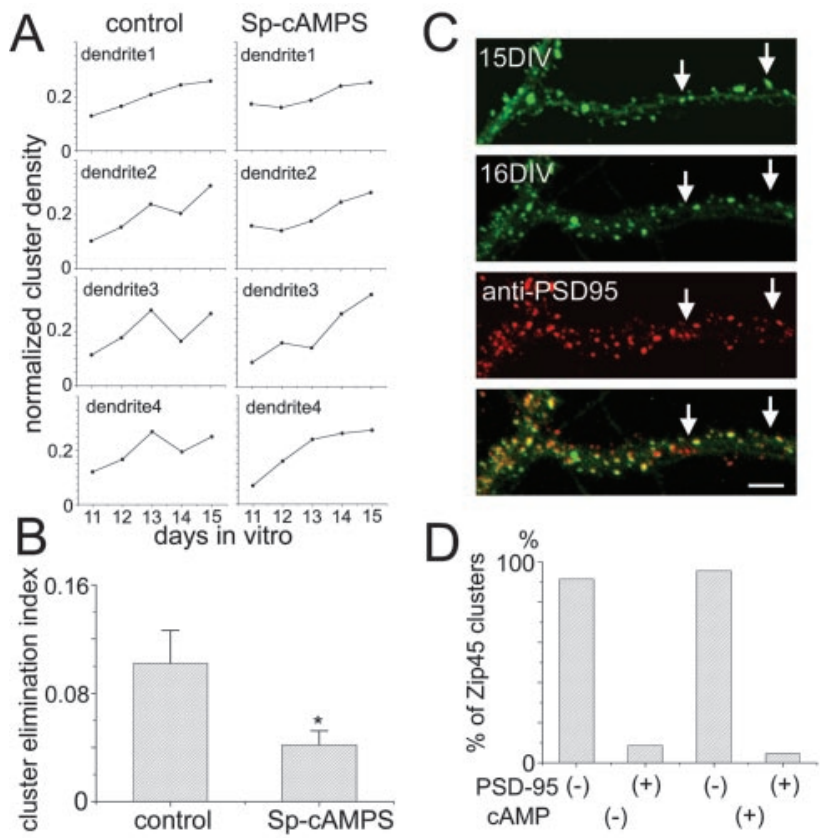

Figure 11. Suppression of GFP-PSD-Zip45 cluster elimination by activation of CAMPdependent signaling mechanisms. A, Patterns of cluster density change in four dendritic segments of a single neuron. Cells were either treated with $100 \mu \mathrm{m}$ Sp-cAMPS from 11 to $15 \mathrm{~d}$ in culture or unperturbed. The temporal profiles of the control neurons show decremental phases of cluster remodeling. The decremental phases were less prominent in neurons treated with Sp-cAMPS. B, The average cluster elimination index (the cumulative number of eliminated clusters divided by the sum of the cluster numbers; see Materials and Methods) was significantly reduced in cells treated with Sp-cAMPS. Data are derived from analysis of a total of 12 dendritic segments in four neurons for both control and treated culture preparations $\left({ }^{*} p<0.05\right)$. Two independent culture preparations showed similar effects of Sp-CAMPS (suppression of cluster elimination indexes were 41.1 and $41.4 \%$ of the controls). C, Retrospective immunocytochemistry of GFP-PSD-Zip45 expressing neurons with anti-PSD-95 antibody. After recording of the GFP-fluorescence from 11 to $16 \mathrm{~d}$ in culture (DIV), cells were fixed and immunostained with anti-PSD-95 antibody. The temporal patterns of cluster remodeling were analyzed for each dendritic segment, and the segments in the decremental phase were analyzed for the presence of PSD-95 clusters at the dendritic domains where GFP-PSD-Zip45 clusters disappeared (arrows). Scale bar, $5 \mu \mathrm{m}$. D, Absence of PSD-95 clusters in the dendritic domains that had lost GFP-PSD-Zip45 clusters. More than $90 \%$ of the dendritic domains were immunonegative with anti-PSD-95 antibody both in the absence and presence of Sp-CAMPS.

odium was subsequently stabilized to form a stable spine. Improvement of the culturing technique of primary neurons on a microscope stage will be important to monitor the whole stage of the spine development.

Analysis of GFP-PSD-Zip45 and PSD-95-GFP clusters revealed that regulation of the cluster addition and elimination was controlled on a single-cell level. This finding suggests the presence of a signaling mechanism that controls the total number of synapses within a single neuron. This mechanism should cooperate with the other mechanisms, such as long-term potentiation, that control the strength of individual synapses to produce the synapse heterogeneity. It is possible that inhibitory molecules present throughout dendrites regulate the balance between assembly and disassembly of PSD molecules. In the case of PSDZip45, Homer 1a is an ideal molecule to play this role, because this protein is induced by neuronal activity and competes with Homer 1c (PSD-Zip45) for its binding partners (Xiao et al., 1998). Dynamics of PSD-95 can be potentially regulated by reversible palmitoylation of the two Cys residues at the N-terminal domain (El-Husseini et al., 2002). It is possible that dense synaptic contacts promote activation of AMPA receptors and subse- 


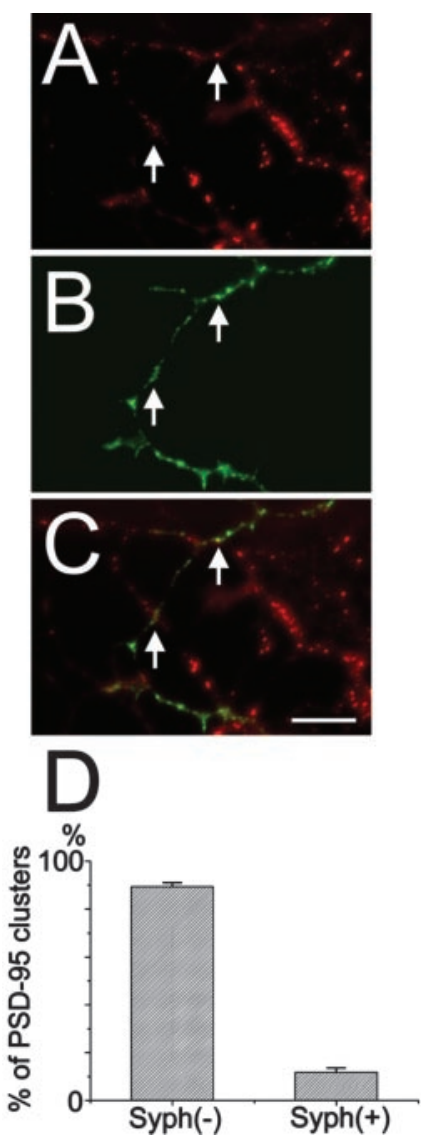

Figure 12. Identification of the PSD structures associated with a single afferent fiber. $A-C$, Fluorescence images of the dendritic field expressing PSD-95-YFP $(A$, red) and a single incoming axon expressing synaptophysin-CFP ( $B$, green). The contact sites between these two fluorescent clusters can be identified in the superimposed image ( $C$, arrows). $D$, The fraction of PSD-95 clusters in a single postsynaptic cell making contact with synaptophysin puncta derived from a single axon (Syph $(+))$ was only $11.7 \%$ of the total PSD-95 clusters. In turn, the fraction of PSD-95 clusters without presynaptic fluorescent clusters (Syph $(-))$, possibly associated with other axons, was $88.3 \%$. Data were derived from analysis of a total of 10 PSD-95-YFPexpressing neurons in two independent culture preparations. Scale bar, $10 \mu \mathrm{m}$.

quently lead to the PSD-95 depalmitoylation, which has been shown to disperse synaptic PSD-95 clusters. Because PSD-95 and PSD-Zip45 are known to be involved in the formation of the molecular complex that links NMDA receptors and mGluRs, activation of NMDA receptors and mGluRs is a possible trigger of PSD assembly and disassembly. It has been shown previously that activation of NMDA receptors and mGluRs can induce structural change of synapses (Engert and Bonhoeffer, 1999; MaleticSavatic et al., 1999; Vanderklish and Edelman, 2002). Possible involvement of these two glutamate receptors in the synchronized PSD remodeling should be clarified by identifying the experimental conditions to selectively manipulate the activity of receptors.

Phosphorylation is another candidate means for regulating synchronized remodeling of the PSD proteins. Previous studies showed that suppression of PKA inhibited potentiation of previously depressed synapses (Kameyama et al., 1998; Lee et al., 2000). PKA activation can recruit NMDA receptors to the synapses (Rao and Craig, 1997; Crump et al., 2001). Furthermore, a recent study showed postsynaptic targeting of PKA and A-kinase anchoring protein 79/150 and their dynamic redistribution (Gomez et al., 2002). Therefore, it is possible that activation of PKA protects PSDs from elimination through the potentiation of the depressed synapses. Our observation that activation of cAMPdependent signaling mechanisms prevented the cells from entering the decremental phase of PSD-Zip45 cluster formation is consistent with this hypothesis. It is also possible that other signaling mechanisms, such as the protein kinase $\mathrm{C}$ pathway, are involved in the molecular dynamics of the PSD. Activation of protein kinase $\mathrm{C}$ induces accumulation of Homer la but not Homer 1c at the synaptic sites (Kato et al., 2001). The accumulated Homer 1a can suppress the formation of PSD-Zip45 clusters. In this case, protein kinase $\mathrm{C}$ activation and activitydependent synthesis of Homer la should be coupled to influence the PSD-Zip45 remodeling.

A large body of work has suggested that suppression of synaptic activity can enhance the synaptic strength (Rao and Craig, 1997; Lissin et al., 1998; Turrigiano et al., 1998; Liao et al., 1999). This process, called disuse hypersensitivity, is associated with an increase in synaptic size, including the average area of the PSDs (Murthy et al., 2001). In our culture system, cells containing a higher density of PSDs are likely to be the cells receiving more excitatory inputs. The synchronized elimination of PSDs during long-term synaptic remodeling can be explained as a reversal of disuse hypersensitivity; namely, the cells receiving inadequately strong inputs can use the synchronized PSD elimination to set the synaptic strength constant. Our experimental data suggest the possibility that synchronized PSD elimination plays a role in homeostatic regulation of total synaptic strength within a single cell.

\section{References}

Ahmari SE, Buchanan J, Smith SJ (2000) Assembly of presynaptic active zones from cytoplasmic transport packets [see comments]. Nat Neurosci 3:445-451.

Brakeman PR, Lanahan AA, O’Brien R, Roche K, Barnes CA, Huganir RL, Worley PF (1997) Homer: a protein that selectively binds metabotropic glutamate receptors. Nature 386:284-288.

Crump FT, Dillman KS, Craig AM (2001) cAMP-dependent protein kinase mediates activity-regulated synaptic targeting of NMDA receptors. J Neurosci 21:5079-5088.

El-Husseini AE, Schnell E, Chetkovich DM, Nicoll RA, Bredt DS (2000) PSD-95 involvement in maturation of excitatory synapses. Science 290:1364-1368.

El-Husseini AE, Schnell E, Dakoji S, Sweeney N, Zhou Q, Prange O, GauthierCampbell C, Aguilera-Moreno A, Nicoll RA, Bredt DS (2002) Synaptic strength regulated by palmitate cycling on PSD-95. Cell 108:849-863.

Engert F, Bonhoeffer T (1999) Dendritic spine changes associated with hippocampal long-term synaptic plasticity. Nature 399:66-70.

Gomez LL, Alam S, Smith KE, Horne E, Dell'Acqua ML (2002) Regulation of A-kinase anchoring protein 79/150-cAMP-dependent protein kinase postsynaptic targeting by NMDA receptor activation of calcineurin and remodeling of dendritic actin. J Neurosci 22:7027-7044.

Harris KM, Jensen FE, Tsao B (1992) Three-dimensional structure of dendritic spines and synapses in rat hippocampus (CA1) at postnatal day 15 and adult ages: implications for the maturation of synaptic physiology and long-term potentiation. J Neurosci 12:2685-2705.

Hayashi Y, Shi SH, Esteban JA, Piccini A, Poncer JC, Malinow R (2000) Driving AMPA receptors into synapses by LTP and CaMKII: requirement for GluR1 and PDZ domain interaction. Science 287:2262-2267.

Kameyama K, Lee HK, Bear MF, Huganir RL (1998) Involvement of a postsynaptic protein kinase A substrate in the expression of homosynaptic long-term depression. Neuron 21:1163-1175.

Kanegae Y, Makimura M, Saito I (1994) A simple and efficient method for purification of infectious recombinant adenovirus. Jpn J Med Sci Biol 47:157-166.

Kanegae Y, Lee G, Sato Y, Tanaka M, Nakai M, Sakaki T, Sugano S, Saito I (1995) Efficient gene activation in mammalian cells by using recombinant adenovirus expressing site-specific Cre recombinase. Nucleic Acids Res 23:3816-3821.

Kato A, Ozawa F, Saitoh Y, Fukazawa Y, Sugiyama H, Inokuchi K (1998) Novel members of the Vesl/Homer family of PDZ proteins that bind metabotropic glutamate receptors. J Biol Chem 273:23969-23975. 
Kato A, Fukuda T, Fukazawa Y, Isojima Y, Fujitani K, Inokuchi K, Sugiyama H (2001) Phorbol esters promote postsynaptic accumulation of Vesl1S/Homer-1a protein. Eur J Neurosci 13:1292-1302.

Kennedy MB (2000) Signal-processing machines at the postsynaptic density. Science 290:750-754.

Kim JH, Huganir RL (1999) Organization and regulation of proteins at synapses. Curr Opin Cell Biol 11:243-257.

Lee HK, Barbarosie M, Kameyama K, Bear MF, Huganir RL (2000) Regulation of distinct AMPA receptor phosphorylation sites during bidirectional synaptic plasticity. Nature 405:955-959.

Liao D, Zhang X, O’Brien R, Ehlers MD, Huganir RL (1999) Regulation of morphological postsynaptic silent synapses in developing hippocampal neurons. Nat Neurosci 2:37-43.

Lissin DV, Gomperts SN, Carroll RC, Christine CW, Kalman D, Kitamura M, Hardy S, Nicoll RA, Malenka RC, von Zastrow M (1998) Activity differentially regulates the surface expression of synaptic AMPA and NMDA glutamate receptors. Proc Natl Acad Sci USA 95:7097-7102.

Lissin DV, Carroll RC, Nicoll RA, Malenka RC, von Zastrow M (1999) Rapid, activation-induced redistribution of ionotropic glutamate receptors in cultured hippocampal neurons. J Neurosci 19:1263-1272.

Maletic-Savatic M, Malinow R, Svoboda K (1999) Rapid dendritic morphogenesis in CA1 hippocampal dendrites induced by synaptic activity. Science 283:1923-1927.

Miyake S, Makimura M, Kanegae Y, Harada S, Sato Y, Takamori K, Tokuda C, Saito I (1996) Efficient generation of recombinant adenoviruses using adenovirus DNA- terminal protein complex and a cosmid bearing the full-length virus genome. Proc Natl Acad Sci USA 93:1320-1324.

Murthy VN, Schikorski T, Stevens CF, Zhu Y (2001) Inactivity produces increases in neurotransmitter release and synapse size. Neuron 32:673-682.

Okabe S, Kim HD, Miwa A, Kuriu T, Okado H (1999) Continual remodeling of postsynaptic density and its regulation by synaptic activity. Nat Neurosci 2:804-811.

Okabe S, Miwa A, Okado H (2001a) Spine formation and correlated assembly of presynaptic and postsynaptic molecules. J Neurosci 21:6105-6114.

Okabe S, Urushido T, Konno D, Okado H, Sobue K (2001b) Rapid redistribution of the postsynaptic density protein PSD-Zip45 (Homer 1c) and its differential regulation by NMDA receptors and calcium channels. J Neurosci 21:9561-9571.

Pak DT, Yang S, Rudolph-Correia S, Kim E, Sheng M (2001) Regulation of dendritic spine morphology by SPAR, a PSD-95-associated RapGAP. Neuron 31:289-303.

Rao A, Craig AM (1997) Activity regulates the synaptic localization of the NMDA receptor in hippocampal neurons. Neuron 19:801-812.

Sala C, Piech V, Wilson NR, Passafaro M, Liu G, Sheng M (2001) Regulation of dendritic spine morphology and synaptic function by Shank and Homer. Neuron 31:115-130.

Schwartz IR, Pappas GD, Purpura DP (1968) Fine structure of neurons and synapses in the feline hippocampus during postnatal ontogenesis. Exp Neurol 22:394-407.

Scott EK, Luo L (2001) How do dendrites take their shape? Nat Neurosci 4:359-365.

Shen K, Meyer T (1999) Dynamic control of CaMKII translocation and localization in hippocampal neurons by NMDA receptor stimulation. Science 284:162-166

Sheng M, Sala C (2001) Pdz domains and the organization of supramolecular complexes. Annu Rev Neurosci 24:1-29.

Shi SH, Hayashi Y, Petralia RS, Zaman SH, Wenthold RJ, Svoboda K, Malinow R (1999) Rapid spine delivery and redistribution of AMPA receptors after synaptic NMDA receptor activation. Science 284:1811-1816.

Sun J, Tadokoro S, Imanaka T, Murakami SD, Nakamura M, Kashiwada K, Ko J, Nishida W, Sobue K (1998) Isolation of PSD-Zip45, a novel Homer/vesl family protein containing leucine zipper motifs, from rat brain. FEBS Lett 437:304-308.

Tadokoro S, Tachibana T, Imanaka T, Nishida W, Sobue K (1999) Involvement of unique leucine-zipper motif of PSD-Zip45 (Homer1c/vesl-1L) in group 1 metabotropic glutamate receptor clustering. Proc Natl Acad Sci USA 93:1540-1544.

Turrigiano GG, Leslie KR, Desai NS, Rutherford LC, Nelson SB (1998) Activity-dependent scaling of quantal amplitude in neocortical neurons. Nature 391:892-896.

Vanderklish PW, Edelman GM (2002) Dendritic spines elongate after stimulation of group 1 metabotropic glutamate receptors in cultured hippocampal neurons. Proc Natl Acad Sci USA 99:1639-1644.

Xiao B, Tu JC, Petralia RS, Yuan JP, Doan A, Breder C, Ruggiero A, Lanahan AA, Wenthold RJ, Worley PF (1998) Homer regulates the association of group 1 metabotropic glutamate receptors with multivalent complexes of Homer-related, synaptic proteins. Neuron 21:707-716.

Ziv NE, Smith SJ (1996) Evidence for a role of dendritic filopodia in synaptogenesis and spine formation. Neuron 17:91-102. 